\title{
Hydrogen-oxygen therapy can alleviate radiotherapy-induced hearing loss in patients with nasopharyngeal cancer
}

\author{
Jibing Chen ${ }^{1 \#}$, Xiaofeng Kong ${ }^{1 \#}$, Feng Mu${ }^{1}$, Tianyu $\mathrm{Lu}^{1}$, Duanming Du ${ }^{2}$, Kecheng Xu ${ }^{1}$ \\ ${ }^{1}$ Central Laboratory, Fuda Cancer Hospital of Jinan University, Guangzhou 510665, China; ${ }^{2}$ Intervention Department, Shenzhen Second People's \\ Hospital, Shenzhen 518035, China \\ \#These authors contributed equally to this work. \\ Correspondence to: Kecheng Xu. No.2 of Tangde West Rd., Tianhe District, Fuda Cancer Hospital of Jinan University, Guangzhou 510665, China. \\ Email: xukc@vip.163.com; Duanming Du. Intervention Department of Shenzhen Second People’s Hospital, No. 3002 of Sungang West Rd., Futian \\ District, Shenzhen 518035, China. Email: 13068872049@163.com.
}

\begin{abstract}
Three patients with nasopharyngeal carcinoma developed binaural secretory otitis media 12, 2, and 0.5 years after radiotherapy, respectively. The secretions subsided after conventional drug and drainage treatments, but hearing continued to deteriorate until severe loss was documented in both ears. After examination of the eardrum and tympanum, patients were enrolled in a clinical trial in the first half of 2019 (ClinicalTrials.gov: NCT03818347). After 0.5, 1 and 2 months of continuous hydrogen-oxygen therapy, our first three patients reported different levels of improvement in binaural hearing. This is the first report to show that, after treatment for nasopharyngeal carcinoma, hearing loss can be alleviated using hydrogenoxygen therapy.
\end{abstract}

Keywords: Nasopharyngeal carcinoma; radiotherapy; secretory otitis media; hydrogen-oxygen therapy; tone audiogram

Submitted Sep 19, 2019. Accepted for publication Nov 05, 2019.

doi: 10.21037/apm.2019.11.18

View this article at: http://dx.doi.org/10.21037/apm.2019.11.18

\section{Introduction}

A nasopharyngeal carcinoma is one of the most common cancers of the head and neck in Asian populations. In southern China, the incidence of nasopharyngeal carcinoma is $20-50$ per 100,000 population (1). Radiotherapy is the main treatment for nasopharyngeal carcinoma. However, patients often experience different degrees of adverse reactions after radiotherapy, such as dry mouth, difficulty in swallowing, secretory otitis media (SOM), sinusitis, hearing loss or brain injury (2). Many adverse reactions are permanent and worsen progressively, which can reduce the quality of life of patients severely $(3,4)$. Studies on whether radiotherapy-induced deafness can be alleviated are lacking.

We treated three patients who suffered SOM after radiotherapy for nasopharyngeal carcinoma. Their hearing was normal before radiotherapy, and SOM appeared after radiotherapy. The secretions subsided after drug treatment, but the extent of hearing loss continued to increase, and a hearing aid was needed for 6-15 years. After 1-2 months of treatment with a hydrogen-oxygen nebulizer, the binaural hearing of the three patients showed different levels of improvement, and one of the patients no longer needs a hearing aid.

\section{Case presentation}

\section{Case 1}

A 63-year-old woman was diagnosed with a poorly differentiated, stage-I, nasopharyngeal squamous cell carcinoma in December 2000. The radiotherapy dose was 72 Gy given in 36 fractions. This tumor was diagnosed as being in complete remission after treatment.

In March 2012, tongue-muscle atrophy, unclear speech, reduced hearing, reduced sense of smell, and SOM in both 


\section{A}
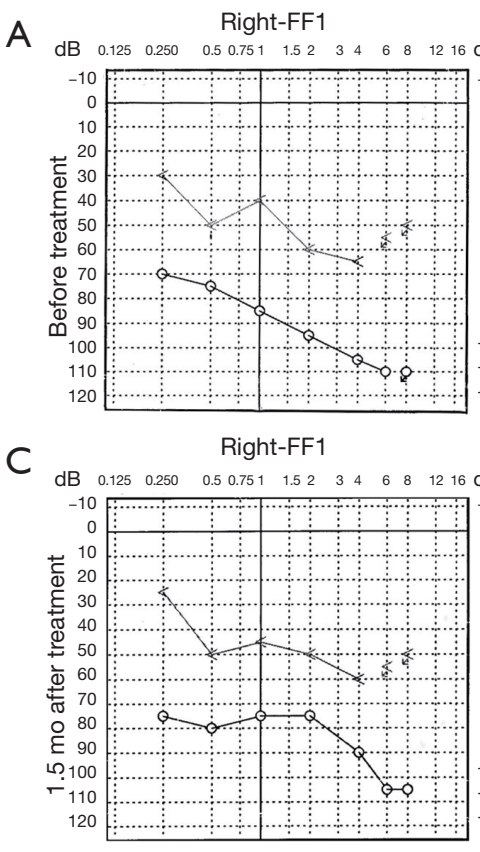

E

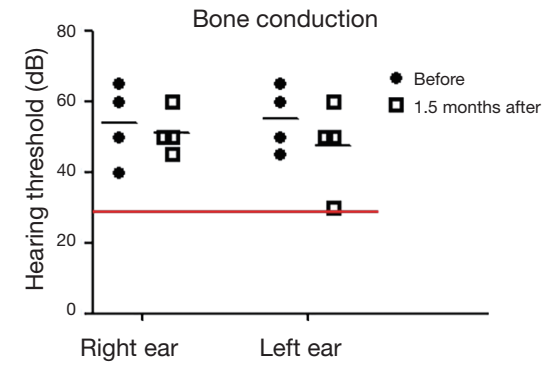

Left-FF2

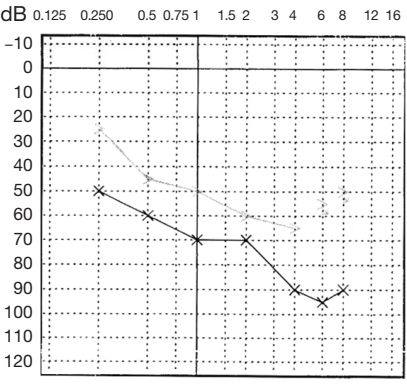

Left-FF2

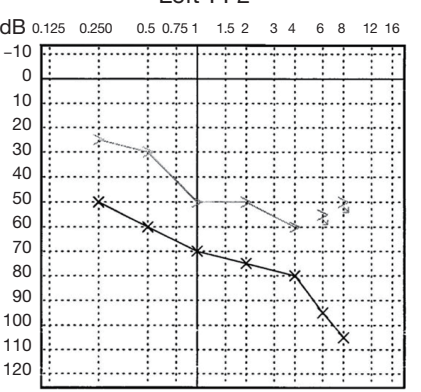

B

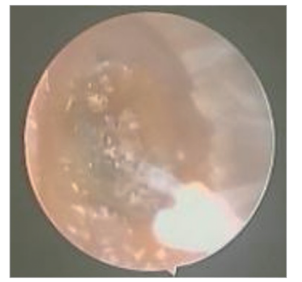

Before treatment

Right

D

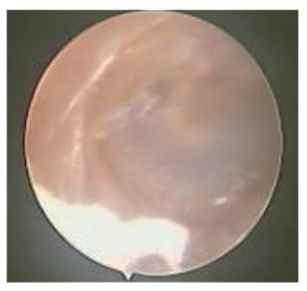

1.5 months after treatment Right

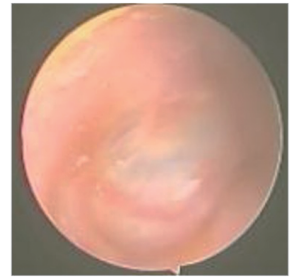

Left

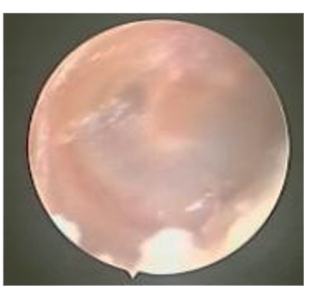

Left

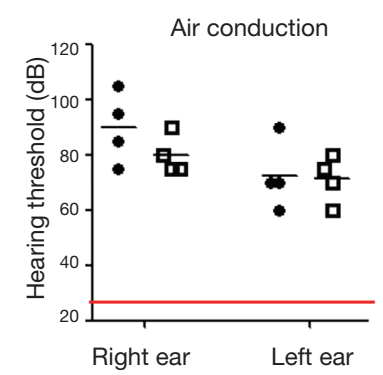

Figure 1 Pure-tone audiograms for Case 1. (A) Hearing tests before enrollment and treatment. The abscissa shows the different sound frequencies. The ordinate shows the hearing threshold at each field frequency (FF). The upper polyline represents bone conduction and the lower represents air conduction. (B) Hearing tests 1.5 months after hydrogen-oxygen treatment. (C) Comparison of the hearing threshold of our patient and normal hearing range. The red line represents the normal hearing range (0-25 $\mathrm{dB})$. (D) Otoscopy results of patient before hydrogen treatment. (E) Otoscopy results 1.5 months after hydrogen treatment.

ears began to appear. After symptomatic treatments, ear fluid and pus disappeared, but hearing loss in both ears was severe, and she started to use a hearing aid.

A pre-treatment check for hydrogen-oxygen therapy (HOT) was undertaken in May 2019. Pure-tone audiometry using an audiometer (AT235; Interacoustics, Copenhagen, Denmark) was done (Figure $1 A$ ) and revealed the decibel $(\mathrm{dB})$ number she could hear under a sound frequency of $500,1,000,2,000$ and 4,000 Hz. The mean hearing threshold (in $\mathrm{dB}$ ) of bone conduction and air conduction was 53.75 and 90, and that of the left ear was 55 and 72.5 , respectively, which denoted severe hearing loss in both ears.

A tympanogram at $226 \mathrm{~Hz}$ using a tympanometer (AT22t; Interacoustics) showed a tympanic B-type map for binaural hearing, which suggested effusion and perforation of the tympanic membrane.

Otoscopy revealed thickening of the right tympanic membrane without a cone of light, healing of scars of bilateral tympanic membranes, and no obvious secretions in the external auditory canal (Figure 1B).

The patient underwent treatment with a hydrogenoxygen nebulizer (AMS-H-03; Asclepius Meditec, Shanghai, China) for $4 \mathrm{~h}$ each day. This machine generated $3 \mathrm{~L} / \mathrm{min}$ hydrogen gas by the electrolysis of water $(67 \%$ hydrogen and $33 \%$ oxygen). On day-16 of treatment, she began to hear sounds in her left ear. After 1.5 months of treatment, she reported that her binaural hearing had improved considerably. The audiogram showed improvements: the 

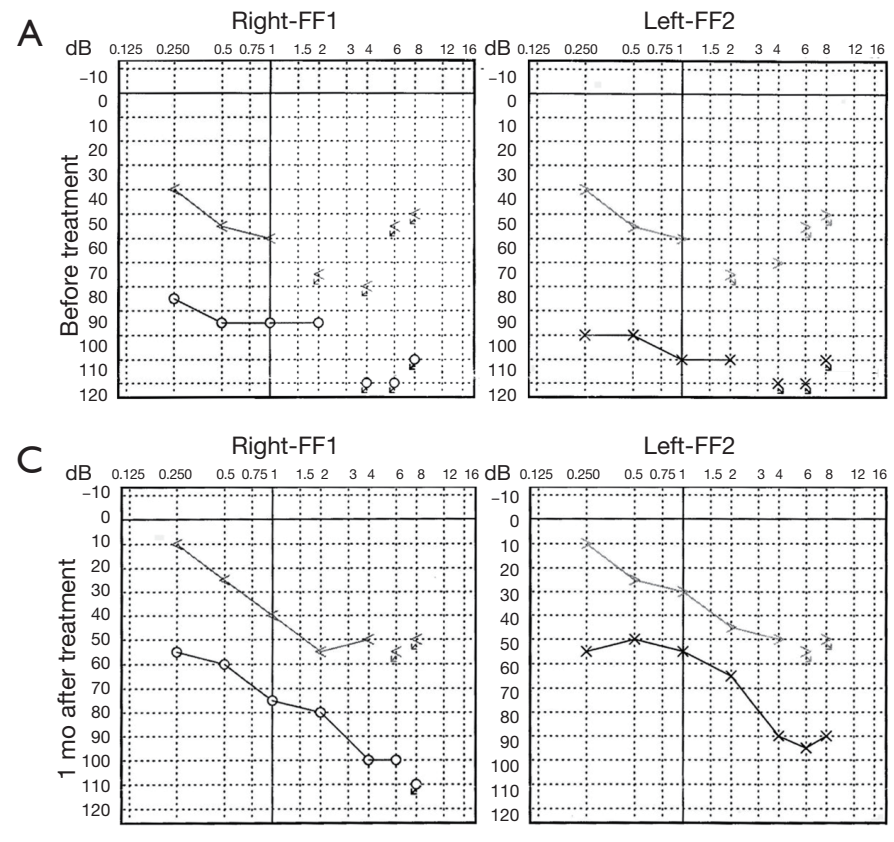

B

D

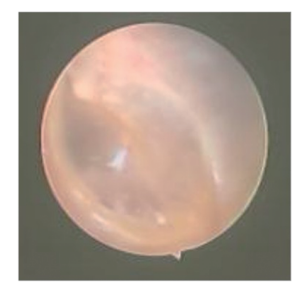

Before treatment

Right

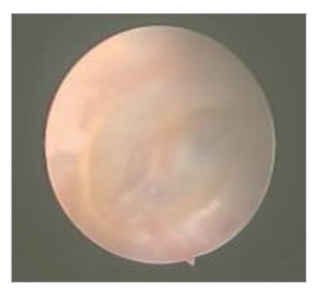

Left

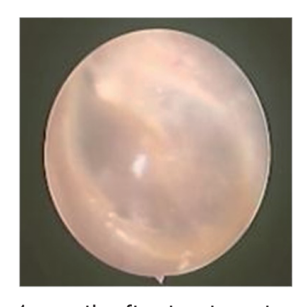

1 month after treatment

Right

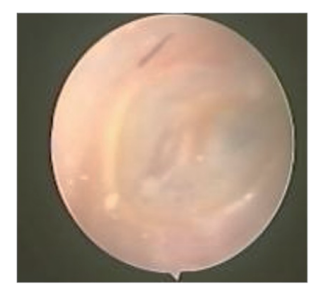

Left
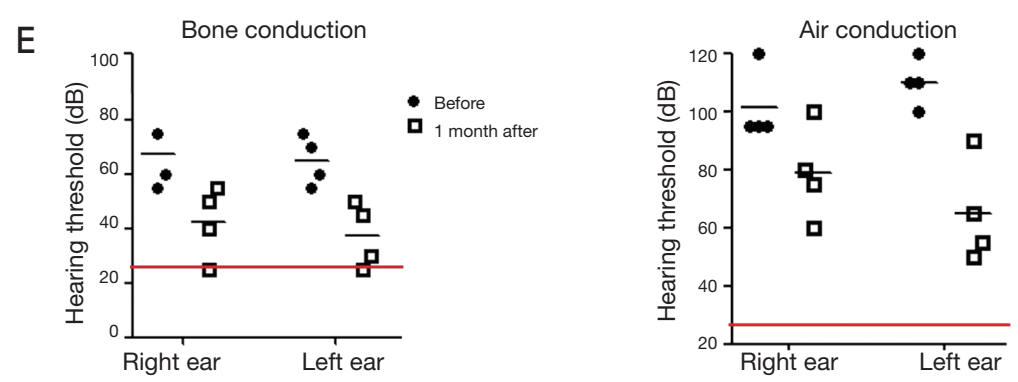

Figure 2 Pure-tone audiograms for Case 2. (A) Hearing tests before enrollment and treatment. The abscissa shows the different sound frequencies. The ordinate shows the hearing threshold at each field frequency (FF). The upper polyline represents bone conduction and the lower represents air conduction. (B) Hearing test 1 month after hydrogen-oxygen treatment. (C) Comparison of the hearing threshold of our patient and normal hearing range. The red line represents the normal hearing range (0-25 dB). (D) Otoscopy results of patient before hydrogen treatment. (E) Otoscopy results 1 month after hydrogen treatment.

mean result (in $\mathrm{dB}$ ) for bone conduction and air conduction in the right ear was 51.25 and 80 , and that of the left ear was 47.5 and 71.25 , respectively (Figure 1C), but no change was observed after tympanometry and otoscopy (Figure 1D). Detection of a pure tone in both ears improved, but it continues to take longer to treat that that for a normal hearing range in humans (Figure 1E).

\section{Case 2}

A 64-year-old women was diagnosed as having a stageII, undifferentiated, non-keratinized nasopharyngeal carcinoma in October 2010. She received radiotherapy
(70 Gy in 36 fractions), and the tumor was judged to be in complete remission. Headache, insomnia, severe dry mouth and bilateral SOM appeared after radiotherapy.

After symptomatic treatments, ear fluid and pus disappeared. However, hearing loss in both ears was severe, and a hearing aid was started in February 2013. A pre-treatment check for hydrogen therapy was undertaken in May 2019.

A pure-tone audiogram is shown as Figure $2 A$, and reveals the $\mathrm{dB}$ number she could hear under a sound frequency of 500, 1,000, 2,000 and 4,000 Hz. The mean hearing threshold (in $\mathrm{dB}$ ) of bone conduction and air conduction in the right ear was 67.5 and 101.25 , and that of the left ear was 65 and 110 , respectively, which denoted 
extremely severe hearing loss in both ears.

A tympanogram at $226 \mathrm{~Hz}$ showed a tympanic B-type map for binaural hearing, suggesting effusion and perforation of the tympanic membrane. Otoscopy revealed that both sides of the tympanic membrane were turbid and invaginated, and that the cone was deformed (Figure 2B).

The patient underwent treatment with a hydrogenoxygen nebulizer for $4 \mathrm{~h}$ each day. After 1 month of treatment, she reported that her binaural hearing had improved considerably. The audiogram showed improvements: the mean value (in $\mathrm{dB}$ ) for bone conduction and air conduction in the right ear was 42.5 and 78.75 , and that of the left ear was 37.5 and 65, respectively (Figure 2C), but no change was observed after tympanometry and otoscopy (Figure 2D). Detection of a pure tone for both ears improved, but it continues to take longer to treat than that for a normal hearing range in humans (Figure 2E).

\section{Case 3}

A 63-year-old man was diagnosed as having a stage-II, poorly differentiated, nasopharyngeal squamous cell carcinoma in January 2000. The radiotherapy dose was 72 Gy given in 40 fractions. This tumor was considered to be in complete remission after treatment.

Hearing loss along with ear pus was noticed first in September 2001, and he underwent ear drainage. Hearing loss increased gradually, and hearing aids have been used since June 2016.

A pre-treatment check for HOT was undertaken in May 2019. A pure-tone audiogram is shown as Figure 3 A, and reveals the $\mathrm{dB}$ number he could hear under a sound frequency of 500, 1,000, 2,000 and 4,000 Hz. The mean hearing threshold (in $\mathrm{dB}$ ) of bone conduction and air conduction in the right ear was 22.5 and 62.5 , and that in the left ear was 25 and 58.75, respectively, which suggested moderate hearing loss in both ears.

A tympanogram at $226 \mathrm{~Hz}$ showed a B-type map for the left tympanum and C-type map for the right tympanum. These data suggested eustachian-tube dysfunction in the right ear, and effusion/large perforation of the tympanic membrane in the left ear. Otoscopy revealed turbidity and thickening of bilateral tympanic membranes (Figure 3B).

We underwent treatment with a hydrogen-oxygen nebulizer for $4 \mathrm{~h}$ each day. After 2 months of treatment, he reported that binaural hearing had improved considerably. The audiogram showed improvements: the mean bone conduction and air conduction (in $\mathrm{dB}$ ) in the right ear was 12.5 and 41.25 , and that of the left ear was 12.5 and 42.5, respectively (Figure 3C), but no change was observed after tympanometry and otoscopy (Figure 3D). The boneconduction hearing of both ears remained normal, and airconduction hearing continued to improve, so the patient stopped using a hearing aid (Figure 3E).

\section{Discussion}

A nasopharyngeal carcinoma, and its main treatment, radiotherapy, can cause conductive hearing loss due to external- and middle-ear damage and sensorineural hearing loss due to injury to the cochlea and auditory nerves (2). The main parts of the middle ear to be damaged are the tympanic membrane, tympanic mucosa, and the eustachian tube, and the clinical manifestation is SOM (2). Otitis media caused by tumor compression can usually be alleviated completely after radiotherapy without causing sustained hearing loss. Usually, inflammation caused by radiotherapy does not appear immediately after treatment, but instead appears suddenly after many years, and causes hearing loss (and even deafness) rapidly (5). About half of patients with a nasopharyngeal carcinoma have SOM by 5 years following radiotherapy completion (5).

Our three patients developed SOM suddenly 12, 2, and 0.5 years after radiotherapy. After conventional treatment, hearing loss disappeared gradually, which suggested postirradiation hearing loss. There is no specific treatment for inner-ear injury and hearing loss caused by radiotherapy for nasopharyngeal carcinoma. We reported, for the first time, that the hearing loss caused by radiotherapy for nasopharyngeal carcinoma can be alleviated, and that use of hearing aids can be stopped.

The three patients reported here were the first to show improvement in clinical features and pure-tone audiometry. Although the time to hearing loss after radiotherapy was very different $(12,2$, and 0.5 years, respectively), the time to considerable improvement was similar (1.5, 1 , and 2 months, respectively), indicating that there was no correlation between these two factors. The degree of hearing loss in the high-frequency region $(4,000 \mathrm{~Hz})$ that Honore and co-workers described in 2002 was correlated positively with the radiation dose given to the cochlea, the age of the patient, and hearing status before radiotherapy (6). The factors mentioned above were similar for our three patients. Hence, the reason why hydrogen therapy could alleviate hearing loss may be related to these factors.

Interestingly, our three patients with self-reported 

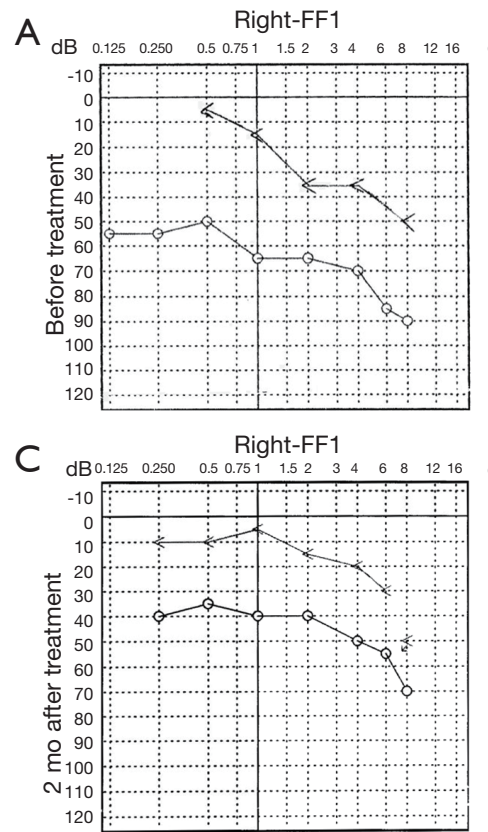

$E$

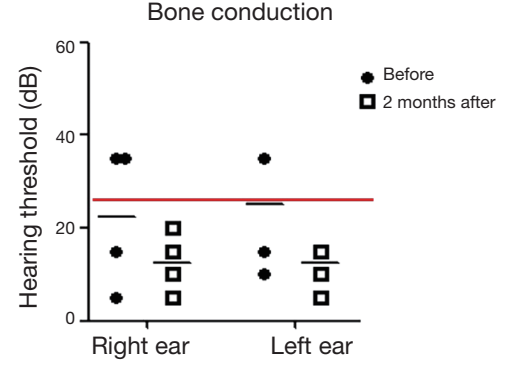

Left-FF2

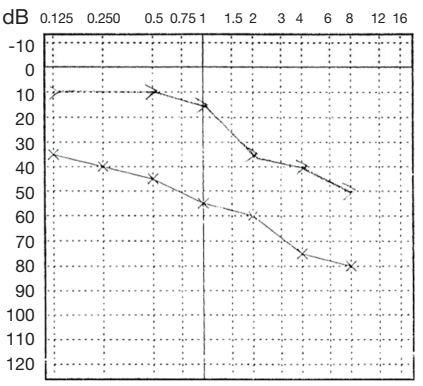

Left-FF2

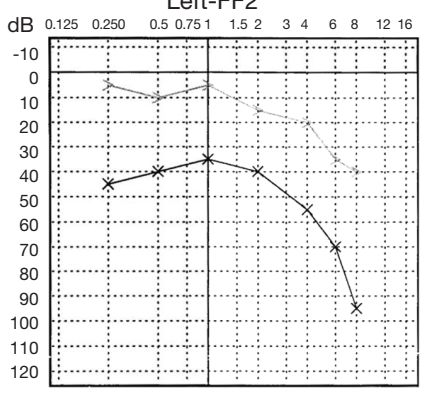

B

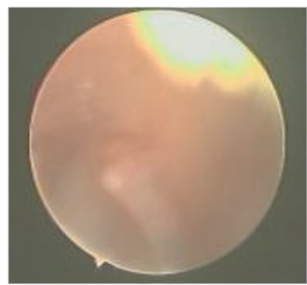

Before treatment

Right

$\mathrm{D}$

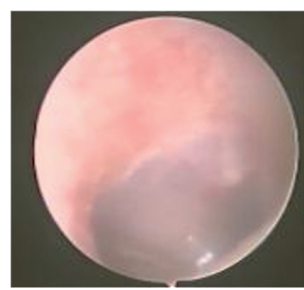

2 months after treatment

Right

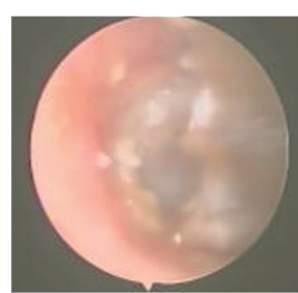

Left

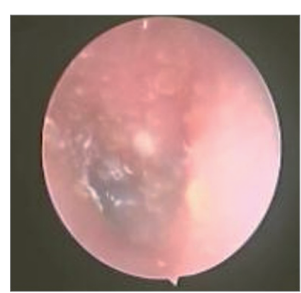

Left

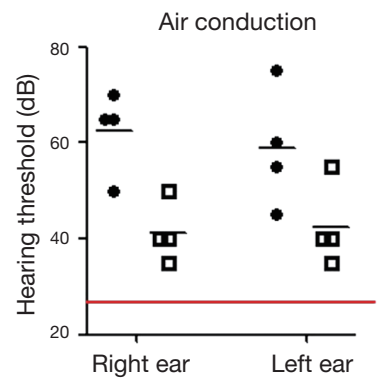

Figure 3 Pure-tone audiograms for Case 3. (A) Hearing test before enrollment and treatment. The abscissa shows the different sound frequencies. The ordinate shows the hearing threshold at each field frequency (FF). The upper polyline represents bone conduction and the lower represents air conduction. (B) Hearing test 2 months after hydrogen-oxygen treatment. (C) Comparison of the hearing threshold of our patient and normal hearing range. The red line represents the normal hearing range (0-25 $\mathrm{dB})$. (D) Otoscopy results of patient before hydrogen treatment. (E) Otoscopy results 2 months after hydrogen treatment.

hearing improvement had only improved pure-tone audiograms, whereas the tympanograms and otoscopy data did not change, suggesting that this improvement was functional rather than organic. Therefore, whether such improvement can be maintained for a long-time merits further study. Case 3 was able to stop using hearing aids for normal communication, and we postulate that the puretone audiogram can be used as an independent prognostic factor to evaluate treatment outcomes.

In 2007, Ohsawa et al. were the first to report that hydrogen has antioxidant effects, and its biologic effects have received increasing attention since then (7). Studies have shown that hydrogen has: (I) selective anti-oxidation, anti-inflammatory and anti-apoptotic effects (8); (II) good therapeutic effects in 70 disease models; (III) achieved good results in clinical trials. Some lesions are associated with oxidative stress, the inflammatory response and local-tissue hypoxia (9).

In our three patients, after HOT, the pus in the ear disappeared, and the threshold of air conduction and bone conduction decreased considerably, which may have been related to the anti-oxidation and anti-inflammatory effects of the hydrogen-oxygen mixture. Studies have suggested that the levels of hypoxia-inducible transcription factors increase during hypoxia, then increase the expression of multiple signaling pathways and molecules, and neutrophil 
activation increases levels of superoxide anions and reactive oxygen species (10). Hydrogen can relieve chronic inflammation by antagonizing these mechanisms (11). Studies have demonstrated that hyperbaric oxygen can improve the therapeutic effect of sudden deafness and promote hearing recovery (12). Therefore, treatment with a mixture of hydrogen and oxygen can improve local hypoxia, reduce inflammation, reduce oxidative stress, and aid cell repair.

The hearing threshold of a patient during pure-tone audiometry reflects the condition of the cochlea. The hearing threshold of our patients was reduced considerably. Improvement in hearing upon HOT could be related to improvement of injury to the cochlea. One advantage of HOT at sites in the head and neck compared with those at other sites is the short distance needed for its diffusion, which leads to high concentrations during HOT.

\section{Conclusions}

The radiotherapy-induced hearing loss in the three patients in our study was relieved after HOT, but the mechanism of action is not clear. Further research is needed to provide safe, economical and efficacious treatment for patients with hearing loss after radiotherapy for nasopharyngeal carcinoma.

\section{Acknowledgments}

None.

\section{Footnote}

Conflicts of Interest: The authors have no conflicts of interest to declare.

Ethical Statement: The authors are accountable for all aspects of the work in ensuring that questions related to the accuracy or integrity of any part of the work are appropriately investigated and resolved. Written informed consent was obtained from the patients for publication of this manuscript and any accompanying images.

\section{References}

1. Li C, Wang Y. Factors associated with early diagnosis in pediatric vs adult nasopharyngeal carcinoma. Acta
Otolaryngol 2018;138:56-9.

2. Sun D, Zhao M, Yin J, et al. Vestibular function disorders and potential mechanisms in irradiation nasopharyngeal carcinoma patients. Acta Otolaryngol 2016;136:759-63.

3. Tian YM, Huang WZ, Yuan X, et al. The challenge in treating locally recurrent T3-4 nasopharyngeal carcinoma: the survival benefit and severe late toxicities of re-irradiation with intensity-modulated radiotherapy. Oncotarget 2017;8:43450-7.

4. Kong F, Zhou J, Du C, et al. Long-term survival and late complications of intensity-modulated radiotherapy for recurrent nasopharyngeal carcinoma. BMC Cancer 2018;18:1139.

5. Hsin CH, Tseng HC, Lin HP, et al. Post-irradiation otitis media, rhinosinusitis, and their interrelationship in nasopharyngeal carcinoma patients treated by IMRT. Eur Arch Otorhinolaryngol 2016;273:471-7.

6. Honoré HB, Bentzen SM, Møller K, et al. Sensorineural hearing loss after radiotherapy for nasopharyngeal carcinoma: individualized risk estimation. Radiother Oncol 2002;65:9-16.

7. Ohsawa I, Ishikawa $M$, Takahashi $\mathrm{K}$, et al. Hydrogen acts as a therapeutic antioxidant by selectively reducing cytotoxic oxygen radicals. Nat Med 2007;13:688-94.

8. Ohta S. Molecular hydrogen as a novel antioxidant: overview of the advantages of hydrogen for medical applications. Methods Enzymol 2015;555:289-317.

9. Nishiguchi $M$, Ono $S$, Iseda $K$, et al. Effect of vasodilation by milrinone, a phosphodiesterase III inhibitor, on vasospastic arteries after a subarachnoid hemorrhage in vitro and in vivo: effectiveness of cisternal injection of milrinone. Neurosurgery 2010;66:158-64; discussion 64.

10. Thompson AA, Binham J, Plant T, et al. Hypoxia, the HIF pathway and neutrophilic inflammatory responses. Biol Chem 2013;394:471-7.

11. Wang YZ, Li TT, Cao HL, et al. Recent advances in the neuroprotective effects of medical gases. Med Gas Res 2019;9:80-7.

12. Gaitanou K, Fildissis G, Vavasis P, et al. Management of sudden hearing loss with hyperbaric oxygen therapy. Undersea Hyperb Med 2014;41:363-70.

Cite this article as: Chen J, Kong X, Mu F, Lu T, Du D, Xu K Hydrogen-oxygen therapy can alleviate radiotherapy-induced hearing loss in patients with nasopharyngeal cancer. Ann Palliat Med 2019;8(5):746-751. doi: 10.21037/apm.2019.11.18 